SHORT REPORT

\title{
Application of the FICTION technique for the simultaneous detection of immunophenotype and chromosomal abnormalities in routinely fixed, paraffin wax embedded bone marrow trephines
}

\author{
P Korać, M Jones, M Dominis, R Kušec, D Y Mason, A H Banham, R A Ventura
}

J Clin Pathol 2005;58:1336-1338. doi: 10.1136/icp.2005.026468

\begin{abstract}
The use of interphase fluorescence in situ hybridisation (FISH) to study cytogenetic abnormalities in routinely fixed paraffin wax embedded tissue has become commonplace over the past decade. However, very few studies have applied FISH to routinely fixed bone marrow trephines (BMTs). This may be because of the acid based decalcification methods that are commonly used during the processing of BMTs, which may adversely affect the suitability of the sample for FISH analysis. For the first time, this report describes the simultaneous application of FISH and immunofluorescent staining (the FICTION technique) to formalin fixed, EDTA decalcified and paraffin wax embedded BMTs. This technique allows the direct correlation of genetic abnormalities to immunophenotype, and therefore will be particularly useful for the identification of genetic abnormalities in specific tumour cells present in BMTs. The application of this to routine clinical practice will assist diagnosis and the detection of minimal residual disease.
\end{abstract}

$\mathrm{T}$ he aberrant changes in protein expression, function, and localisation seen in haematological malignancies are often caused by chromosomal abnormalities. ${ }^{1}$ Although the detection of chromosome abnormalities using fluorescence in situ hybridisation (FISH) assays may aid the diagnosis of several types of haematological malignancyfor example, $t(11 ; 14)$ in mantle cell lymphoma (MCL) and $\mathrm{t}(14 ; 18)$ in follicular lymphoma (FL) - it has not been widely applied to the study of bone marrow trephines (BMTs). Since the introduction of EDTA as a decalcifying agent, ${ }^{2}$ there have been a few reports of the successful application of interphase FISH for the detection of cytogenetic abnormalities on routinely fixed BMTs. ${ }^{3}{ }^{4}$ However, because BMTs may contain low numbers of infiltrating neoplastic cells, the identification of abnormal cells using FISH can be very difficult. We have further developed these previous studies and here we report, for the first time, the successful application of a modified FICTION technique to allow the study of BMTs. This technique allows the rapid identification of rare CD20 positive B cells within the bone marrow by immunophenotyping and can simultaneously identify any specific genetic aberrations harboured by these cells.

\section{METHODS}

\section{Tissues}

BMTs from patients diagnosed with FL $(\mathrm{n}=5)$, chronic lymphocytic leukaemia (CLL) $(\mathrm{n}=5), \operatorname{MCL}(\mathrm{n}=2)$, acute lymphoblastic leukaemia (ALL) $(\mathrm{n}=3)$, or with no detectable neoplasms $(\mathrm{n}=7)$ were obtained, with ethical permission, from the Merkur University Hospital, Zagreb, Croatia between 1998 and 2004. After biopsy, the BMTs were stored for a minimum of 12 hours in Schaffer's solution ( $50 \mathrm{ml}$ formalin (37-40\%), $50 \mathrm{ml}$ methanol). Decalcification was performed by incubation for 48-72 hours in either Osteosoft (EDTA based; Merck, Hoddesdon, Hertfordshire, $\mathrm{UK}$ ) or Shandon TBD-1 decalcifier ( $\mathrm{HCl}$ based; Thermo Electron Corporation, Basingstoke, Hampshire, UK). Samples were then dehydrated in an alcohol series (70\% ethanol, 96\% ethanol, and absolute ethanol; two hours each), followed by incubation in Shandon xylene substitute (two hours; Thermo Electron Corporation). Tissues were submerged overnight in liquid Paraplast (Thermo Electron Corporation) before embedding in paraffin wax blocks. Tissue sections $(4 \mu \mathrm{m}$ thick) were then cut, mounted on charged slides (Snowcoat $\mathrm{X}$-tra; Surgipath, Richmond, Illinois, USA), and left to dry overnight at $56^{\circ} \mathrm{C}$.

\section{FICTION}

Tissue sections were dewaxed in Citroclear $(3 \times 5$ minutes; HD Supplies, Aylesbury, Buckinghamshire, UK), washed for two minutes in absolute ethanol, and rehydrated in an alcohol series (absolute ethanol, 85\% ethanol, and 70\% ethanol; two minutes each), followed by submersion for two minutes in deionised $\mathrm{H}_{2} \mathrm{O}$. Slides were then transferred to antigen retrieval buffer $\left(50 \mathrm{ml}\right.$ deionised $\mathrm{H}_{2} \mathrm{O}, 1 \mathrm{mM}$ EDTA, $\mathrm{pH} 8$ ) and heated in a Decloaking Chamber digital pressure cooker (Biocare Medical, Hamburg, Germany) at $125^{\circ} \mathrm{C}$ for three minutes before washing in Tris buffered saline ( $100 \mathrm{ml}$ ) for five minutes.

All incubations were performed at room temperature, in the dark, and interspersed with five minute washes in Tris buffered saline. Sections were sequentially incubated for 30 minutes with a mouse monoclonal antibody directed against CD20 (1/20 dilution; DakoCytomation, Glostrup, Denmark), for 45 minutes with biotin conjugated rabbit antimouse immunoglobulin (1/100 dilution; DakoCytomation), and for 45 minutes with a mixture of AlexaFluor 350 conjugated goat antirabbit immunoglobulin and AlexaFluor 350 conjugated streptavidin (both at $1 / 100$ dilution; Molecular Probes, Leiden, the Netherlands). The slides were washed as before, dehydrated in an alcohol series (70\% ethanol, 85\% ethanol, and absolute ethanol; two minutes each), and allowed to air dry in the dark. Dual fusion $\mathrm{t}(11 ; 14), \mathrm{t}(11 ; 18), \mathrm{t}(8 ; 14)$ and breakapart c-MYC FISH probes (Abbott Diagnostics,

Abbreviations: ALL, acute lymphoblastic leukaemia; BMT, bone marrow trephine; CLL, chronic lymphocytic leukaemia; FISH, fluorescence in situ hybridisation; FL, follicular lymphoma; $\mathrm{MCL}$, mantle cell lymphoma 
Maidenhead, Berkshire, UK) were prepared according to the manufacturer's instructions. An aliquot $(1.6 \mu \mathrm{l})$ was applied to the area of interest on the BMT, covered with a $10 \mathrm{~mm}$ diameter circular coverslip (Raymond Lamb, Eastbourne, East Sussex, UK), and sealed with liquid rubber cement (bicycle tyre repair rubber solution). When the rubber cement had dried, slides were placed in a HYBrite Hybridiser (Abbott Diagnostics) and denatured at $85^{\circ} \mathrm{C}$ for five minutes, followed by overnight hybridisation at $37^{\circ} \mathrm{C}$. The next day, slides were washed three times (five minutes each) in preheated $0.1 \times$ saline sodium citrate at $60^{\circ} \mathrm{C}$, mounted using Vectashield mounting medium for fluorescence (without DAPI) (Vector Laboratories Inc, Burlingame, California, USA), and viewed using a fluorescence microscope equipped with a dual pass fluorescein isothiocyanate/rhodamine filter and an ultraviolet longpass filter (Chroma Technology Corp, Rockingham, Vermont, USA).

\section{RESULTS}

The FICTION technique was applied to BMTs that had been decalcified with either an ETDA based or an $\mathrm{HCl}$ based reagent. When FICTION was performed on acid decalcified BMTs from patients diagnosed with FL $(\mathrm{n}=1)$, CLL $(\mathrm{n}=1), \operatorname{MCL}(\mathrm{n}=1)$, and those with no detectable neoplasms $(\mathrm{n}=3)$ there was adequate immunostaining but no FISH signals.

In contrast, the EDTA based treatment enabled the successful application of combined immunohistochemistry and FISH using the FICTION technique described above (fig 1). This technique was applied to BMTs prepared using the EDTA based reagent from patients with no detectable neoplasms $(\mathrm{n}=4)$ and those with FL $(\mathrm{n}=4)$, CLL $(\mathrm{n}=4)$, ALL $(\mathrm{n}=3)$, and MCL $(\mathrm{n}=1)$. The FISH signals in each of the cases were bright, focused, and easily interpretable using a $\times 63$ objective lens, enabling the

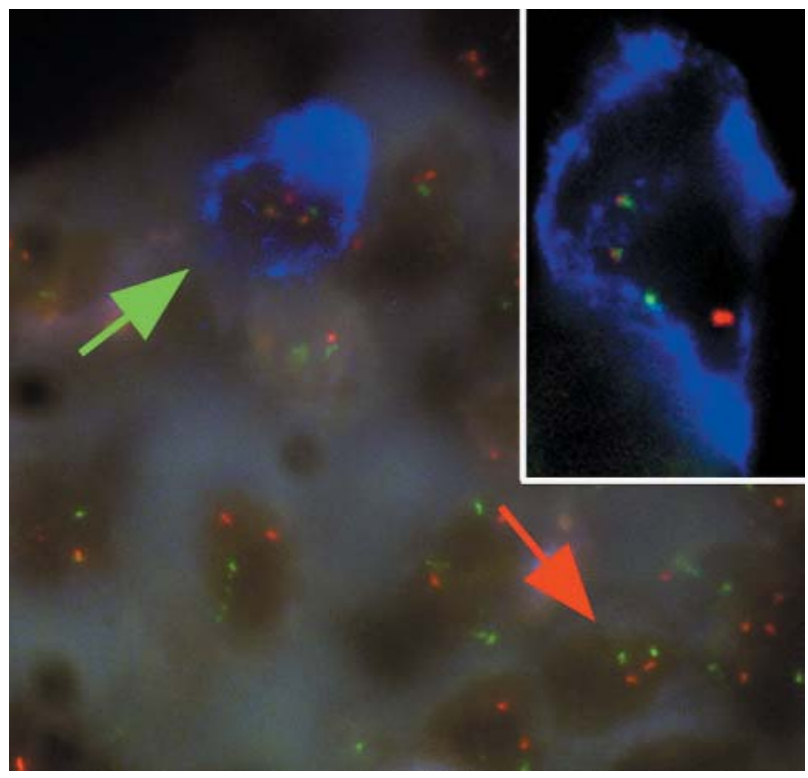

Figure 1 FICTION analysis of a mantle cell lymphoma bone marrow biopsy prepared using EDTA based Osteosoft. Bone marrow sections were immunostained with an anti-CD20 antibody and AlexaFluor 350 conjugated antibodies and streptavidin. Dual fusion fluorescence in situ hybridisation (FISH) probes for the detection of $t(11 ; 14)(13 q ; 32 q)$ were then applied. The blue immunostaining indicates CD20 positive cells and the red and green signals indicate the CCNDI and IGH loci, respectively. Normal cells show two red and two green FISH signals (red arrow), whereas abnormal cells harbouring a CCNDI/IGH translocation show one red, one green, and two fused FISH signals (green arrow and inset).

\section{Take home messages}

- We have developed a technique for the simultaneous application of FISH and immunofluorescent staining (the FICTION technique) to formalin fixed, EDTA decalcified and paraffin wax embedded bone marrow trephines (BMTs)

- This technique allows the direct correlation of genetic abnormalities to immunophenotype, and will be particularly useful for the identification of genetic abnormalities in specific tumour cells present in BMTs

- The application of this technique in routine clinical practice will assist diagnosis and the detection of minimal residual disease

detection of a $t(11 ; 14)$ in the MCL case, a $t(14 ; 18)$ in the FCL cases, and both a $t(8 ; 14)$ and a split at the $c-M Y C$ locus in the ALL cases. No $t(11 ; 14)$ was detected in the CLL cases. The immunofluorescent staining in each sample was bright, specific, did not fade, and facilitated the rapid identification of neoplastic cells.

\section{DISCUSSION}

Several groups have confirmed the value and effectiveness of evaluating BMTs for the presence of neoplastic B cell infiltrates and for diagnosing B cell malignancies. ${ }^{5-7}$ The determination of bone marrow involvement is also considered in the staging of patients with lymphoma, for monitoring disease progression, assessing the efficacy of treatment regimens, and for identifying minimal residual disease. The identification of neoplastic cells in the bone marrow is of particular importance if a patient is being considered for a stem cell or bone marrow harvest before transplantation.

\section{"This technique is a suitable tool for identifying different neoplasms in bone marrow trephines"}

To the best of our knowledge, our study is the first to apply the FICTION technique to routinely fixed BMTs. Several different commercially available probe sets were used in our study, demonstrating the suitability of this technique as a tool for identifying different neoplasms in BMTs. The FICTION methodology described here (without pressure cooking) is also suitable for use on a wide range of tissue types (including frozen sections, blood smears, touch preparations) and is robust, rapid, and technically easy to perform. The preservation of tissue morphology was better in the EDTA treated samples than in the $\mathrm{HCl}$ treated samples, indicating that EDTA based reagents may also be more suitable for routinely preparing BMTs for immunohistochemistry. In summary, the FICTION technique described here will prove to be a useful tool in research and in a clinical setting for the investigation of CD20 positive cells in BMTs.

\section{ACKNOWLEDGEMENTS}

This work was supported by the Leukaemia Research Fund and PK was supported during this project by a grant from The British Scholarship Trust. The authors wish to thank MM Vrsalović for helpful discussions.

\section{Authors' affiliations}

M Jones, D Y Mason, A H Banham, R A Ventura, Nuffield Department of Clinical Laboratory Sciences, University of Oxford, LRF Immunodiagnostics Unit, John Radcliffe Hospital, Headington, Oxford OX3 9DU, UK 
P Korać, M Dominis, Department of Clinical Pathology and Cytology, Merkur University Hospital, 10000, Zagreb, Croatia

R Kušec, Institute of Clinical Chemistry, Merkur University Hospital

Correspondence to: Dr R Ventura, Nuffield Department of Clinical Laboratory Sciences, University of Oxford, LRF Immunodiagnostics Unit, Level 5, Room 5501, John Radcliffe Hospital, Headington, Oxford OX3 9DU, UK; roland.ventura@ndcls.ox.ac.uk

Accepted for publication 4 April 2005

\section{REFERENCES}

1 Falini B, Mason DY. Proteins encoded by genes involved in chromosomal alterations in lymphoma and leukaemia: clinical value of their detection by immunocytochemistry. Blood 2002;99:409-26.
2 Alers JC, Krijtenburg PJ, Vissers KJ, et al. Effect of bone decalcification procedures on DNA in situ hybridization and comparative genomic hybridization: EDTA is highly preferable to a routinely used acid decalcifier $J$ Histochem Cytochem 1999:47:703-9.

3 Le Maitre CL, Byers RJ, Liu Yin JA, et al. Dual colour FISH in paraffin wax embedded bone trephines for identification of numerical and structural chromosomal abnormalities in acute myeloid leukaemia and myelodysplasia. J Clin Pathol 2001;54:730-3.

4 Brown RS, Edwards J, Bartlett JW, et al. Routine acid decalcification of bone marrow samples can preserve DNA for FISH and CGH studies in metastatic prostate cancer. J Histochem Cytochem 2002;50:113-15.

5 Maes B, Achten R, Demunter A, et al. Evaluation of B cell lymphoid infiltrates in bone marrow biopsies by morphology, immunohistochemistry, and molecular analysis. J Clin Pathol 2000:53:835-40.

6 Pezzella F, Munson PJ, Miller KD, et al. The diagnosis of low-grade peripheral B-cell neoplasms in bone marrow trephines. Br J Haematol 2000; 108:369-76.

7 Buhr T, Langer F, Schlue J, et al. Reliability of lymphoma classification in bone marrow trephines. Br J Haematol 2002;118:470-6. 